\title{
Quantification of proliferative activity in colorectal adenomas by mitotic counts: relationship to degree of dysplasia and histological type
}

\author{
G A Meijer, J P A Baak
}

\begin{abstract}
Aim-Proliferative activity of tumours reflects their malignant potential. In colorectal adenomas, a subjective impression of the number of mitoses is a criterion often used to assess the degree of dysplasia. Since these subjective impressions of $\mathrm{mi}$ totic activity may lack reproducibility, the aim of this study was to perform an objective analysis.

Methods-Mitotic counts were conducted in tissue sections of 59 colorectal adenomas. Of these, 20 showed mild, 20 moderate, and 19 severe dysplasia, according to blind duplicate assessments by two pathologists. Forty three were classified as tubular adenomas and 16 as "villous" adenomas (tubulo-villous and villous). The number of mitoses, both per unit area of epithelium (area weighted mitotic counts, AWMC) and per colonic crypt (mitotic counts per colonic crypt MCCC), was scored in the most dysplastic area within the adenoma. Mitotic figures were counted using a light microscope (ocular $\times 10$, objective $\times 40$, NA 0.75 ), and the area of the glandular epithelium was measured using an interactive video overlay measurement system. Twenty glands per specimen were assessed. In the intra-observer reproducibility tests, the coefficients of error
\end{abstract}

Pepartment of

University Hospital

NL-1081 HV

Amsterdam,

G A Meijer

J P A Baak

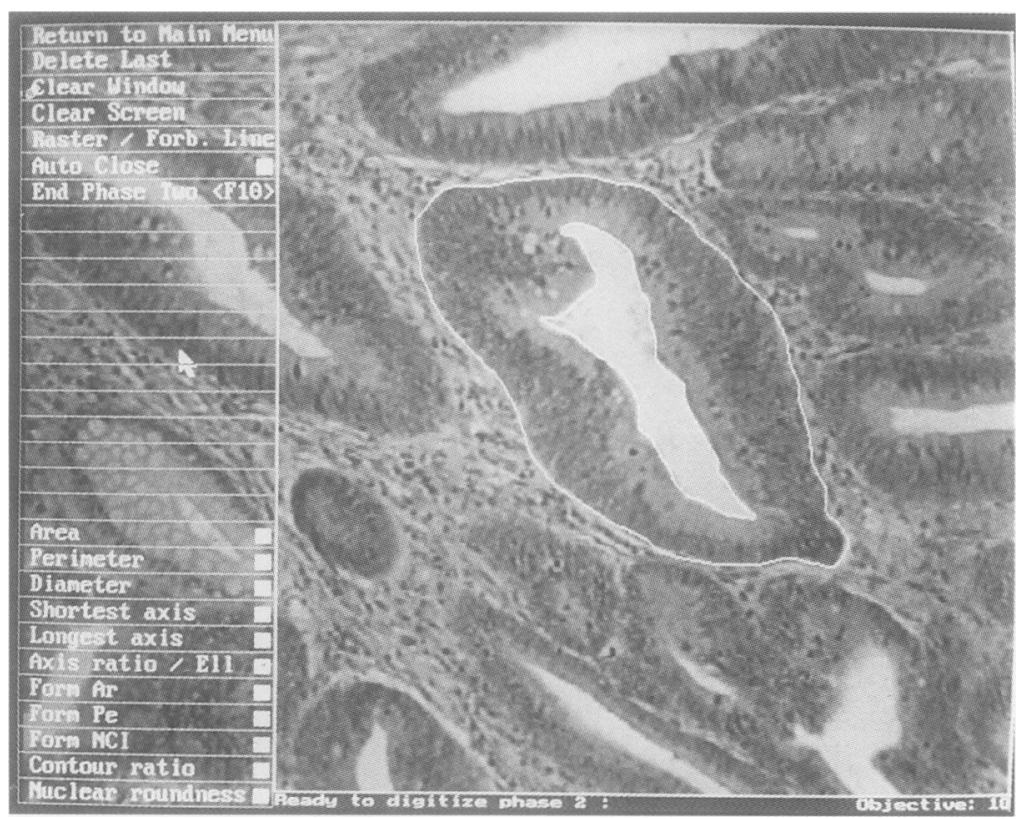

Figure 1 Profile area of colonic crypt epithelium outlined with the PRODIT video overlay measurement system. for the AWMC and MCCC were $4 \cdot 5 \%$ and $\mathbf{7 \cdot 4 \%}$ respectively.

Results-For the AWMC a significant difference was found between mild and moderate as well as between mild and severe dysplasia, but not between moderate and severe dysplasia. The results of the MCCC showed the same trend, but the differences did not reach a significant level. Furthermore, cases classified as mild dysplasia were found that showed numerous mitoses, while cases classified as severe dysplasia were found with only very few mitoses. No significant difference in AWMC was found between tubular and villous adenomas. Thus the different malignant potential of tubular and villous adenomas was not reflected by a difference in AWMC. A seemingly strong difference for MCCC between tubular and "villous" adenomas appeared to depend completely on the difference in crypt size between these two groups.

Conclusions-The area weighted mitotic count, rather than the mitotic count per colonic crypt, may be useful for assessing the proliferation rate in colorectal adenomas.

(f Clin Pathol 1995;48:620-625)

Keywords: Colorectal adenoma, mitosis, proliferation.

The management of adenomatous polyps of the large intestine plays an important role in the secondary prevention of colorectal cancer, the second leading cause of cancer related death in the western world. Patients in whom colorectal adenomas have been removed are, because of their assumed high risk of future colorectal carcinoma, submitted to an intensive follow up programme which includes repeated colonoscopies. However, this increased risk goes only for a minority of these patients. ${ }^{1}$ Identification of this specific subgroup at risk would enable more specific, and therefore more (cost-)effective strategies, in order to prevent colorectal cancer from occurring. This is even more important against the background of preventive population screening strategies. ${ }^{2} \mathrm{Be}$ cause of the high prevalence of colorectal adenomas in patients over 60 years, these screening strategies would yield a very large number of patients to be taken under surveillance, thereby tremendously increasing the cost of such screening strategies. ${ }^{34}$ In this regard, it is important that several observations 
Means and 95\% confidence intervals (CI) of the area weighted mitotic counts (AWMC) and number of mitotic counts per colonic crypt (MCCC) in colorectal adenomatous polyps grouped by grade of dysplasia and histological type

\begin{tabular}{|c|c|c|c|c|c|c|c|c|c|c|}
\hline & \multicolumn{6}{|c|}{ Dysplasia } & \multicolumn{4}{|c|}{ Histological type } \\
\hline & \multicolumn{2}{|l|}{ Mild } & \multicolumn{2}{|c|}{ Moderate } & \multicolumn{2}{|c|}{ Severe } & \multicolumn{2}{|c|}{ Tubular } & \multicolumn{2}{|c|}{ Villous } \\
\hline & mean & $95 \% C I$ & mean & $95 \% C I$ & mean & $95 \% C I$ & mean & $95 \% C I$ & mean & $95 \% C I$ \\
\hline $\begin{array}{l}\text { AWMC }\left(\mathrm{No} / 10000 \mu \mathrm{m}^{2}\right) \\
\operatorname{MCCC}(\mathrm{No} / \mathrm{crypt})\end{array}$ & $\begin{array}{l}0 \cdot 67 \\
2 \cdot 22\end{array}$ & $\begin{array}{l}0.54-0.79 \\
1.51-2.93\end{array}$ & $\begin{array}{l}0.99 \\
3 \cdot 34\end{array}$ & $\begin{array}{l}0 \cdot 76-1 \cdot 23 \\
2 \cdot 26-4 \cdot 41\end{array}$ & $\begin{array}{l}0.99 \\
3 \cdot 44\end{array}$ & $\begin{array}{l}0 \cdot 80-1 \cdot 19 \\
2 \cdot 34-4 \cdot 54\end{array}$ & $\begin{array}{l}0 \cdot 89 \\
2 \cdot 54\end{array}$ & $\begin{array}{l}0 \cdot 76-1 \cdot 02 \\
2 \cdot 13-2 \cdot 94\end{array}$ & $\begin{array}{l}0 \cdot 86 \\
4 \cdot 21\end{array}$ & $\begin{array}{l}0.64-1.09 \\
2 \cdot 51-5.91\end{array}$ \\
\hline
\end{tabular}

have indicated that the morphology of adenomas could provide valuable prognostic information. ${ }^{5-7}$ Classically, adenoma histology is classified subjectively as grade of dysplasia and histological type. To overcome difficulties inherent to subjective classification systems, ob-
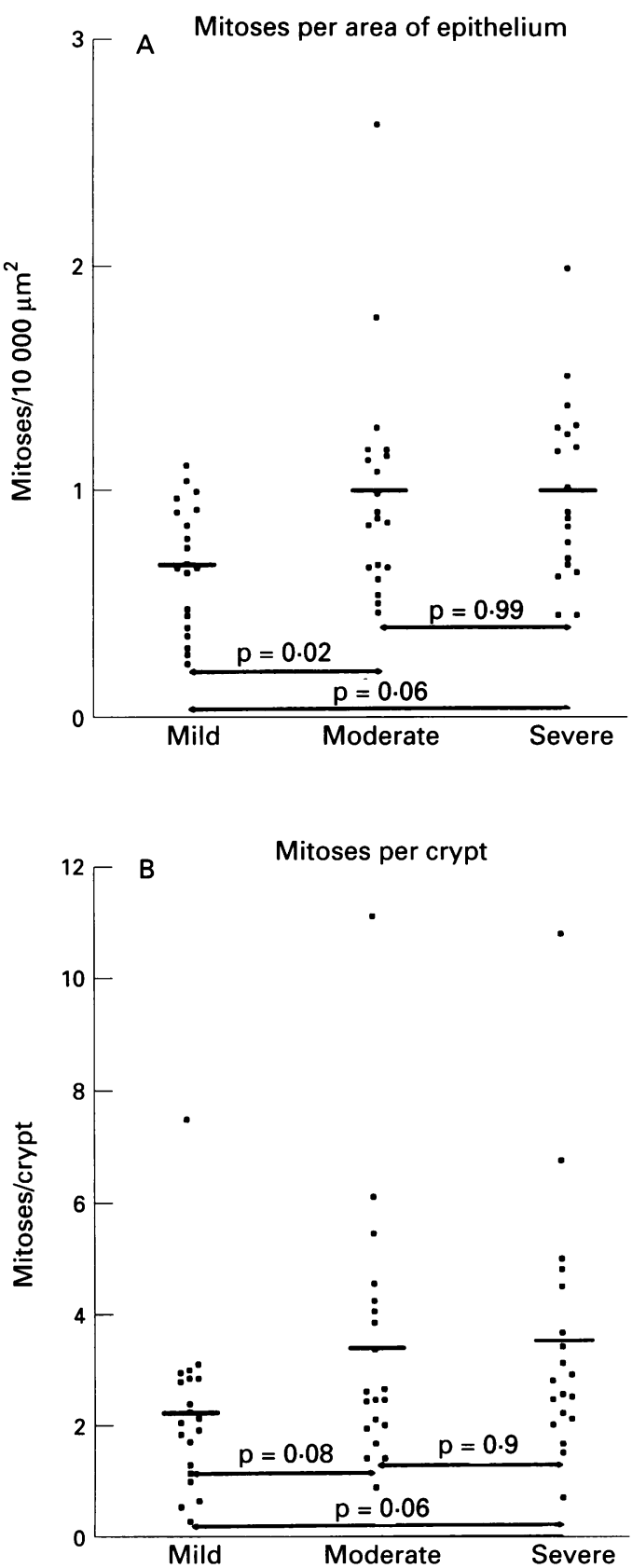

Figure 2 A significant difference in the number of mitoses per unit of area (area weighted mitotic counts $=A W M C$, left) was found between mild and moderate dysplasia as well as between mild and severe dysplasia, but not between moderate and severe dysplasia, in 59 colorectal adenomas.

The mitotic counts per colonic crypt (MCCC, right) showed the same trend, although significance was not reached. jective classifiers are being developed, which may help to distinguish cases at high risk of colorectal cancer more reliably. ${ }^{8-10}$

In addition to judging nuclear atypia and glandular changes, the subjective appraisal of the number of mitoses, which gives an impression of the proliferative activity, is one of the criteria used in grading dysplasia in colorectal adenomas. ${ }^{112}$ Moreover, proliferative activity is generally regarded as a marker of malignant biological potential. Several of the genetic events that are supposed to play a role in the pathogenesis of colorectal cancer, like k-ras and p53 mutations, probably directly affect the control of cell proliferation. ${ }^{1314}$

An efficient method for assessing proliferative activity is to count mitoses. ${ }^{15}$ Despite criticism as to a possible lack of reproducibility, it has been shown that the method can be highly reproducible provided a strict counting protocol is used. ${ }^{16}$ Furthermore, the influence of delayed fixation ${ }^{17}$ probably does not play an important role in the case of colorectal adenomas, since in general the biopsy or polypectomy specimen is stored immediately in fixative. In this study we aimed to investigate whether the quantitatively assessed number of mitoses correlated with the subjectively assessed degree of dysplasia. Furthermore, we were interested to see if adenomas of different histological type, comprising a different risk of malignancy, would differ in mitotic rate.

\section{Methods}

PATIENT MATERIAL

Specimens of colorectal adenomatous polyps were obtained from the archives of our institute. The tissue had been fixed in mercury-formalin $(\mathrm{pH}=2 \cdot 2)$, embedded in paraffin, cut at $5 \mu \mathrm{m}$ thickness, and stained with haematoxylineosin. In order to obtain comparable confidence intervals in the statistical analysis, an approximately equal number of adenomas with mild (20 cases), moderate (20 cases), and severe dysplasia (19 cases) was used. From a large number of adenomas, only those cases were selected in which consensus had been reached by two pathologists in a double blind examination. The grade of dysplasia was determined subjectively, using the following generally accepted criteria. ${ }^{12}$ In mild dysplasia the nuclei are elongated, enlarged, slightly hyperchromatic, and crowded, with early stratification. There is little branching of crypts. Moderate dysplasia shows increased nuclear stratification, with more hyperchromaticity, loss of polarity, pleomorphism and mucin depletion. In severe dysplasia, nuclear atypia can be stronger than in moderate dysplasia, with prominent nucleoli and an increased number of 


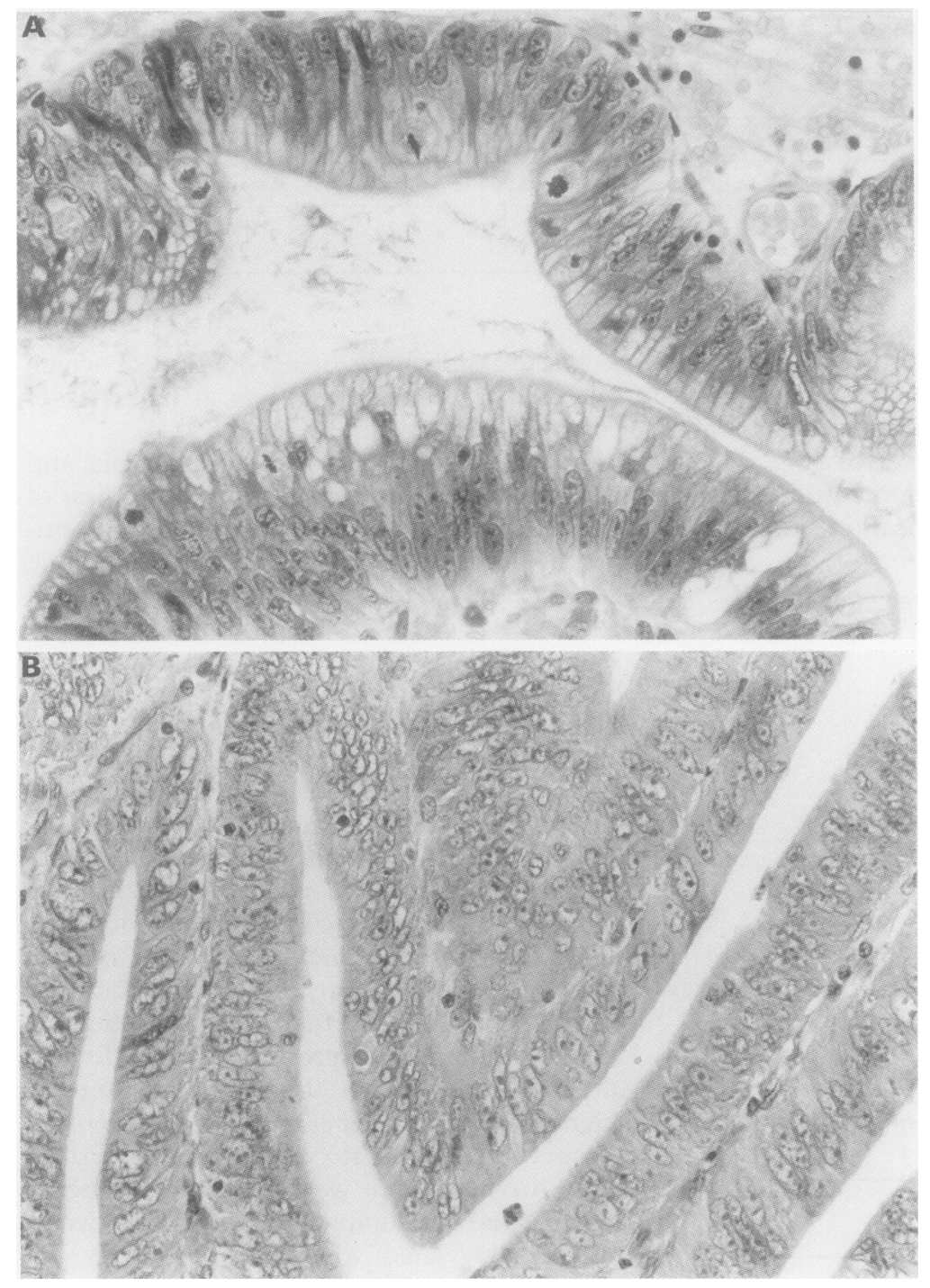

Figure 3 Top: Adenoma with mild dysplasia; nuclei are elongated, slightly enlarged, and hyperchromatic, and there is still ample mucin present. Numerous mitoses are seen. Bottom: Adenoma with severe dysplasia: The nuclei are large, hyperchromatic, atypical, and show prominent nucleoli. There is clear stratification and loss of polarity, pleomorphism and mucin depletion. However, no mitoses are seen.

mitoses. The crypt architecture shows budding, branching, and "back to back" orientation of the glands. Within a single specimen there often is a gradual change in the degree of dysplasia. Therefore the classification of grade was based on the most affected part.

Concerning the histological type, leaf-like or finger-like processes in the two dimensional sections were regarded as representing villous components. ${ }^{18}$ Because of the limited number of cases with villous components, no subdivision was made into tubulovillous and villous adenomas. These cases are therefore further referred to as "villous" adenomas $(n=16)$, which are to be distinguished from the tubular adenomas $(n=43)$.

\section{MEASUREMENTS}

Measurements were performed according to a strict measurement protocol, similar to the ones described earlier. ${ }^{8-10}$ In each histological section a measurement area, usually at the top of the polyp, showing the highest degree of dysplasia and with the highest density of mitoses, was carefully selected and demarcated. Within this field, in 20 adjacent crypts the mitotic figures were counted and the profile area of crypt epithelium was measured.

Mitotic figures were counted using the following well established criteria ${ }^{19}$ : absence of a nuclear membrane; no clear zone in the centre; hairy projections rather than spiky or triangular; and basophilic, amphophilic or pale, but not eosinophilic, cytoplasm. For this purpose we used a light microscope at $\times 400$ magnification (ocular $\times 10$, objective $\times 40$, NA $0 \cdot 75$ ). The area measurements were performed using a commercially available interactive video overlay measuring system (PRODIT version $4 \cdot 1$, BMA, De Meern, The Netherlands). With this system, the microscopical image is recorded by a video camera and displayed on the computer screen. Using a $\times 10$ objective gave a final magnification of $\pm 300 \times$ on the computer screen, which appeared the most suitable for this purpose. Conducting two-phase measurements, first the outer crypt border (that is, the epithelium/stroma border) was demarcated, giving the total crypt area. Second, the inner crypt border (that is, the epithelium/lumen border) was marked, giving the luminal profile area, which then was subtracted from the total crypt area giving the profile area of epithelium (fig 1).

In each specimen, the mean number of mitoses per colonic crypt (MCCC) and the number of mitoses per unit area of epithelium (area weighted mitotic counts, AWMC) were computed. In the intra-observer reproducibility tests, the coefficient of error for the AWMC was $4.5 \%$ and for the MCCC it was $7 \cdot 4 \%$.

\section{STATISTICS}

First, the mean and its $95 \%$ confidence interval were calculated for all three grades of dysplasia, as well as for the tubular and villous cases, both for the MCCC and the AWMC. Second, with the Kruskal-Wallis test with multiple comparisons, the presence of significant differences between the three grades was investigated for both variables, and if present, between which pair of grades they arose. The level of significance was set to $\mathrm{p}<0.05$. The differences between tubular and villous adenomas were assessed with the Mann-Whitney test.

To test the intra-observer reproducibility, three specimens were measured five times. Then the coefficient of error (CE) was calculated over the five measurements per specimen. Averaging these values over the three specimens gave the mean $C E$ for both variables. All analyses were performed with BMDP statistical software.

\section{Results}

The mean values with their $95 \%$ confidence intervals of AWMC and MCCC in mild, moderate, and severe dysplasia are given in the table. For the AWMC a significant difference was found between mild and moderate dysplasia as well as between mild and severe dysplasia, but not between moderate and severe 
dysplasia. The results of the MCCC showed the same trend, but the differences did not reach significance (fig 2). Furthermore, some
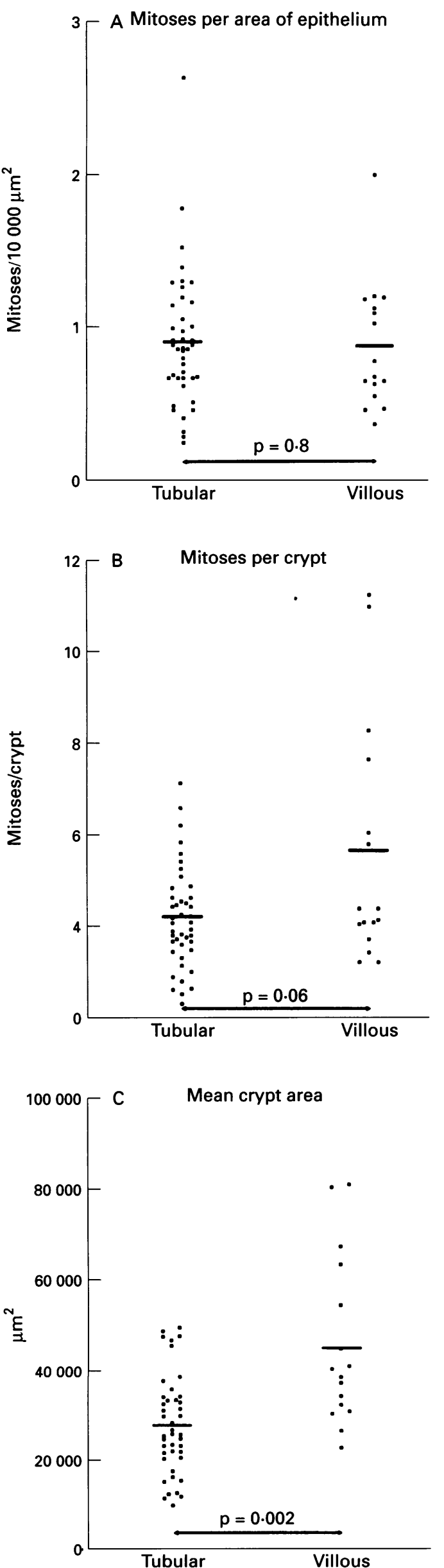

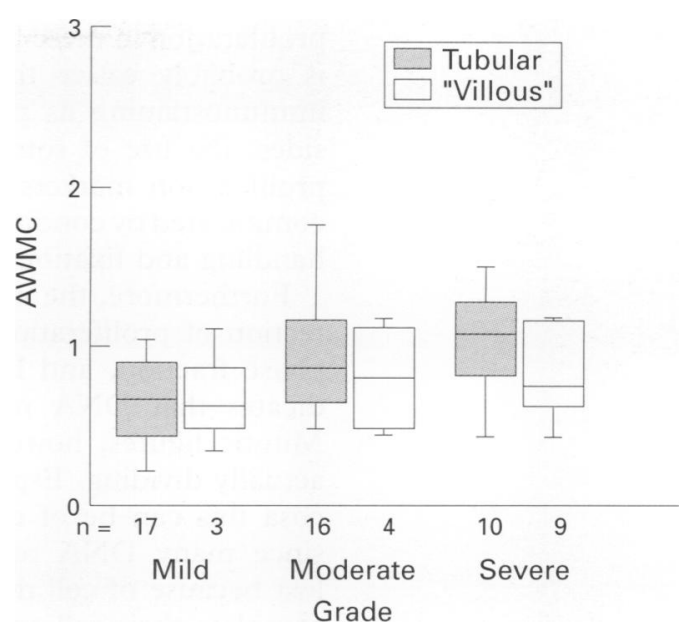

Figure 5 The mean number of mitoses per unit area of epithelium (area weighted mitotic counts $=A W M C$ ) for tubular and "villous" adenomas, stratified after grade of dysplasia.

cases subjectively classified as mild dysplasia showed numerous mitoses, whereas other cases that had been classified subjectively as severe dysplasia showed only a few mitoses (fig 3).

With respect to histological type, the mean values of AWMC and MCCC with their 95\% confidence intervals for tubular and "villous" adenomas are also listed in the table. No significant difference in AWMC was found between the two histological types (fig 4A). This picture persisted after stratification for grade (fig 5). The difference between tubular and "villous" adenomas in MCCC appeared to be entirely caused by the larger crypt size in the latter (fig $4 B$ and $C$ ).

\section{Discussion}

Mitosis counting had been proposed for the classification of colorectal carcinomas as early as $1939,{ }^{20}$ and proliferation rate has been compared in normal colorectal mucosa, adenoma, and carcinoma, using several techniques. ${ }^{21-25}$ Atypical mitoses in colorectal adenomas have recently been studied ${ }^{26}$ but to our knowledge no investigation of the quantification of mitoses in colorectal adenomas with respect to grade of dysplasia and histological type has been performed. This is even more surprising since the subjective assessment of mitotic activity is widely used as one of the criteria for grading dysplasia in colorectal adenomas. ${ }^{112}$

Counting mitoses is one of the oldest techniques for quantifying the dividing fraction, and has clearly shown its value in many areas of tumour pathology. ${ }^{15}$ Furthermore, several studies have shown it to be a highly reproducible technique, provided that the quality of the slides is adequate and a strict protocol for counting the mitoses is used. ${ }^{1627}$ Mitosis counting allows for selective measurement of epithelial proliferation in tissue sections, unlike the $S$ phase fraction derived from DNA flow cytometry. Its directness makes it very suitable for application in routine pathology. Since mitoses in colorectal adenomas are most often clearly visible and easy to identify, it makes mitosis counting the perfect way of assessing 
proliferation in these lesions. Their recognition is probably easier than the classification of immunostaining as positive or negative. Besides, the use of some immunohistochemical proliferation markers like ki67 and PCNA is complicated by conditions with respect to tissue handling and fixation. ${ }^{28}$

Furthermore, the immunohistochemical detection of proliferation linked proteins, the $S$ phase fraction, and labelling indices only indicates that DNA replication is in process. Mitotic figures, however, mark cells that are actually dividing. Especially in colorectal mucosa this can be of considerable importance, since many DNA replicating cells could be lost because of cell desquamation before they complete their cell cycle, and therefore would not contribute to actual tissue proliferation.

Because of the heterogeneity of the epithelium-stroma ratio and of the degree of epithelial dysplasia within colorectal adenomas, we found counting the mitoses per field of vision (the mitotic activity index) unsuitable in this setting. As an alternative we considered the mitotic index (counting mitoses per number of nuclei). This seems very precise at first glance; however, not only is it a laborious method, but a precise count of the number of nuclei in a given colonic crypt is frustrated by the severe overlap of nuclei. Therefore we counted mitoses per unit area of epithelium. In our view, this method allows, at least in colorectal adenomas, a better assessment of the mitotic rate in the area of interest than counting mitoses per field of vision with a correction afterwards for the global volume percentage of epithelium in the adenoma (the volume corrected mitotic index). ${ }^{29}$

Using grade of dysplasia as a standard by which to evaluate the mitotic activity could raise some questions. Indeed, the grading system is highly subjective, and it can hardly be regarded as a gold standard. For this reason we started to evaluate objective measures of the changes in dysplasia of colorectal adenomas in the present study, as well as in some previous studies. ${ }^{8-10}$ Furthermore, the grading system artificially disrupts a biological continuum into three discrete classes, falsely suggesting the existence of three discrete entities. Nevertheless, subjective grading is widely used to describe the changes in dysplasia, and any new method should be compared to it. Moreover, since the results of the present study refer to groups rather than to individual cases, the detection of trends related to the spectrum of changes in dysplasia is possible.

The results of an increase in AWMC as well as the MCCC from mild to moderate dysplasia, but not from moderate to severe dysplasia, parallel the outcome of earlier studies on nuclear morphometry in colorectal adenomas. ${ }^{89}$ This finding suggests that to a certain extent an increase in nuclear atypia in colorectal adenomas correlates with an increase in mitotic activity, which is in accordance with the common grading systems of dysplasia in colorectal adenomas.

The finding of adenomas classified as mild dysplasia but with a high mitotic density on one hand, and adenomas classified as severe dysplasia but with a low mitotic density on the other (see also fig 3), could be explained in two ways. First, these adenomas could simply have been misclassified, since subjective grading does not allow classification of dysplasia with guaranteed precision. Although we cannot exclude the possibility that this could have happened in an individual case, another explanation is also possible. While not losing sight of the limitations of subjective grading, these results could point to the existence of adenomas that share many of the characteristics of mild dysplasia, but which have an increased mitotic activity, as opposed to adenomas sharing features with severe dysplasia, but which have a low density of mitoses.

From the point of view that proliferative activity could be an indication of the (pre-) malignant potential of adenomas, it was somewhat surprising that no difference in AWMC was found between tubular and "villous" adenomas. In fig 5 we show that this result remained the same after stratification for grade of dysplasia. The difference between tubular and "villous" adenomas found for the MCCC appeared to be caused entirely by the larger crypt size in the "villous" adenomas, as was shown in fig $4 \mathrm{C}$.

It has been suggested that the vast majority of colorectal adenomas grow too slowly to become harmful. ${ }^{30}$ As a result, the few adenomas that would actually progress to a carcinoma are expected to show a high mitotic activity, so in a study like this not many of the adenomas investigated would show these features. In view of this, the finding of some cases with very high mitotic activity, irrespective of grade, seems interesting.

In conclusion, this study showed that the area weighted mitotic count in particular seems a useful tool for assessing the proliferation rate in colorectal adenomas. However, the different malignant potential of tubular and villous adenomas was not reflected by a difference in the AWMC

The study was supported by grant No $28 / 1687$ from the Praeventiefonds.

1 Atkin WS, Morson BC, Cuzick J. Long-term risk of colorectal cancer after excision of rectosigmoid adenomas. $N$ Engl F Med 1992;326:658-62.

2 Winawer SJ, O'Brien MJ, Waye JD, Kronborg O, Bond J, Fruhmorgen P, et al. Risk and surveillance of individuals with colorectal polyps. WHO Collaborating Centre for with colorectal polyps. WHO Collaborating Centre for the Prevent

3 Vatn $\mathrm{MH}$, Stalsberg $\mathrm{H}$. The prevalence of polyps of the large intestine in Oslo: an autopsy study. Cancer 1982;49. $819-25$

4 Williams AR, Balasooriya BAW, Day DW. Polyps and cancer of the large bowel: a necropsy study in Liverpool. Gut 1982;23:835-42.

5 Grossman S, Milos ML, Tekawa IS, Jewell NP. Colonoscopic screening of persons with suspected risk factors for colon cancer: II. Past history of colorectal neoplasms. Gastroenterology 1989;96:299-306.

6 Kronborg O, Fenger C. Prognostic evaluation of planned follow-up in patients with colorectal adenomas. An interim report. Int $\mathcal{F}$ Colorectal Dis 1987;2:203-7.

7 Nava H, Carlsson G, Petrelli NJ, Herrera L, Mittelman A Follow-up colonoscopy in patients with colorectal aden Follow-up colonoscopy in patients with colorectal adenomatous polyps. Dis Colon Rectum 1987;30:465-8.

Meijer GA, Baak JP. Cytonuclear morphometry in the assessment of dysplasia in colorectal adenomatous polyps. A pilot study. Pathol Res Pract 1992;188:148-56.

9 Meijer GA, van-Diest PJ, Fleege JC, Baak JP. Syntactic structure analysis of the arrangement of nuclei in dysplastic epithelium of colorectal adenomatous polyps. Anal Quant Cytol Histol 1992;14:491-8. 
10 Meijer GA, Fleege JC, Baak JPA. Stereological assessment of architectural changes in dysplastic epithelium of colorectal adenomatous polyps. Pathol Res Pract 1994;190:333-41.

11 Konishi F, Morson BC. Pathology of colorectal adenomas: a colonoscopic survey. $f$ Clin Pathol 1982;35:830-41

12 Williams GT. Dysplasia in the large intestine. Pathol Res Pract 1985;180:656-64.

13 Wynford-Thomas D. Oncogenes and anti-oncogenes; the molecular basis of tumour behaviour. $\mathcal{F}$ Pathol 1991;165: 187-201.

14 Marx J. How p53 suppresses cell growth. Science 1993;262: $1644-5$.

15 Baak JPA. Mitosis counting in tumors. Hum Pathol 1990; 21:683-5.

16 Diest PJ van, Baak JPA, Matze-Cok P, Wisse-Brekelmans ECM Galen CM van, Kurver PHI, et al. Reproducibility of mitosis counting in 2469 breast cancer specimility results from the multicenter morphometric mammary car-

Donhuijsen K, Schmidt U, Hirche H, Beuningen D van, Budach V. Changes in mitotic rate and cell cycle fractions caused by delayed fixation. Hum Pathol 1990;21:709-14.
Jass JR. Sobin LH. Histological typing of intestinal tumours. Jass JR, Sobin LH. Histological typing of intestinal tumours.
WHO international classification of tumours. Berlin: Springer Verlag, 1989;29-31 (N).

19 Baak JPA, ed. Manual of quantitative pathology in cancer diagnosis and prognosis. Heidelberg: Springer-Verlag, 1990; 169-172.

20 Grinnell RS. The grading and prognosis of carcinoma of the colon and rectum. Ann Surg 1939;109:500-33.

21 Lipkin $M$. Phase 1 and phase 2 proliferative lesions of Cancer 1974;34:878-88.

22 Deschner EE Salmon RJ. Cell kinetics of gastrointestinal cancer and its precursor states. In: DeCosse JJ, Sherlock cancer and its precursor states. In: DeCosse JJ, Sherlock Nijhoff, 1981:1-26.

23 Hammarberg C, Tribukait B, Rubio C, Slezak P. Clinical application of DNA flow cytometry for characterization of colorectal polyps. Acta Endosc 1985;15:265-73.

24 Kanemitsu T, Koike A, Yamamoto S. Study of the cell proliferation kinetics in ulcerative colitis, adenomatous polyps, and cancer. Cancer 1985;56:1094-8.

25 Johnston PG, O'Brien MJ, Dervan PA, Carney DN. Immunohistochemical analysis of cell kinetic parameters in colonic adenocarcinomas, adenomas, and normal mucosa. Hum Pathol 1989;20:696-700.

26 Rubio CA. Atypical mitoses in colorectal adenomas. Pathol Res Pract 1991;187:508-13.

27 Haapasalo H, Collan Y, Montironi R, Pesonen E, Atkin NB. Consistency of quantitative methods in ovarian tumor histopathology. Int $f$ Gynecol Pathol 1990;9:208-16.

28 Rowlands DC, Brown HE, Barber PC, Jones EL. The effect of tissue fixation on immunostaining for proliferating cell nuclear antigen with the monoclonal antibody PC10. $\mathcal{F}$ Pathol 1991;165:356-7.

29 Haapasalo H, Pesonen E, Collan Y. Volume corrected mitotic index (M/V-INDEX). The standard of mitotic activity in neoplasms. Pathol Res Pract 1989;185:551-4.

30 Spratt JS, Spratt JA. Growth rates of benign and malignant neoplasms of the colon. Prog Clin Biol Res 1985;186: 103-20. 